\title{
A Wearable Augmented Reality System Using an IrDA Device and a Passometer
}

\author{
Ryuhei Tenmoku $^{a}$ Masayuki Kanbara $^{a}$ and Naokazu Yokoya ${ }^{a}$ \\ ${ }^{a}$ Graduate School of Information Science, Nara Institute of Science and Technology, \\ 8916-5 Takayama, Ikoma, Nara, 630-0101 Japan
}

\begin{abstract}
This paper describes a wearable augmented reality system with an IrDA device and a passometer. To realize augmented reality systems, the position and orientation of user's viewpoint should be obtained in real time for aligning the real and virtual coordinate systems. In the proposed system, the orientation of user's viewpoint is measured by an inertial sensor attached to the user's glasses, and the position is measured by using an IrDA device and a passometer. First, the user's position is specified exactly when the user comes into the infrared ray range of the IrDA markers which are set up to the appointed points. When the user goes out of the infrared ray range, the user's position is estimated by using a passometer. The passometer is constructed of an electronic compass and acceleration sensors. The former can detect the user's walking direction. The latter can count how many steps the user walks. These data and the user's pace make it possible to estimate the user's position in the neighborhood of the IrDA markers. We have developed a navigation system based on using the techniques above and have proven the feasibility of the system with experiments.
\end{abstract}

Keywords: Wearable Computer, Augmented Reality, IrDA, Passometer

\section{INTRODUCTION}

Since computers have made remarkable progress in resent years, a wearable computer can be realized. ${ }^{1}$ At the same time, Augmented Reality (AR) which merges the real and virtual worlds has received a great deal of attention as a new method for displaying information. ${ }^{2-4}$ If we construct an annotation overlay system for real scene using an AR technique with a wearable computer, it can be applied for many applications. ${ }^{5-9}$ Figure $^{-1}$ 1 shows an example of the annotation overlay system which imposes annotation on the real scene using AR techniques with wearable computers. To realize AR systems, the position and orientation of user's viewpoint are needed for acquiring a relationship between the real and virtual coordinate systems. Usually, Global Positioning System (GPS) is used to measure the user's position outdoors. ${ }^{10}$ In AR systems which use GPS, a differential

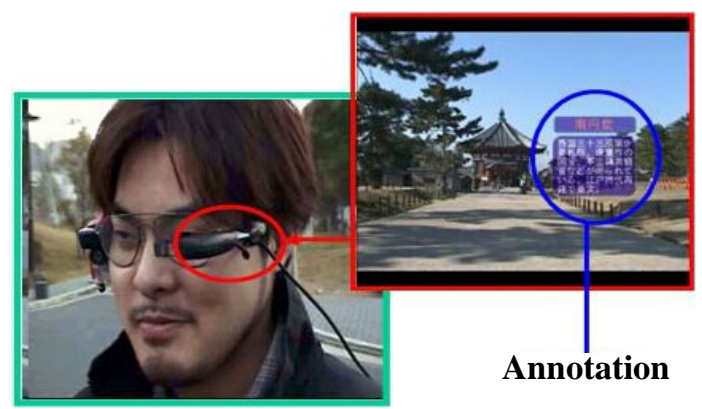

Figure 1. An example of a wearable AR application.

Further author information: (Send correspondence to R.T.)

R.T.: E-mail: ryuhei-t@is.aist-nara.ac.jp, Telephone: +81 743725296

M.K.: E-mail: kanbara@is.aist-nara.ac.jp, Telephone: +81 743725292

N.Y.: E-mail: yokoya@is.aist-nara.ac.jp, Telephone: +81 743725290 
GPS $^{11}$ or a real time kinematic GPS is used to measure the user's position accurately. However, since both of them need radio communication to correct GPS data, the hardware configuration of the system will be complicated. Moreover, GPS cannot be used indoors. Therefore, if we construct a wearable AR system which can be used both indoors and outdoors, we must combine other positioning sensors which can be used indoors such as an electro-magnetic sensor. ${ }^{12}$ Accordingly, the hardware configuration of the system will be more complicated and the system needs to switch the sensors between indoors and outdoors.

The purpose of the present work is to construct a wearable annotation overlay system which can be used both indoors and outdoors seamlessly. We have already proposed the wearable annotation overlay system using RFID tags which can be used at appointed points both of indoors and outdoors. ${ }^{13}$ In this system, RFID tags are set up to appointed points, and the system can identify the user's position reading the RFID tag with a REID tag reader equipped by the user. However, the system needs the user's action bringing an RFID tag reader to an REID tag to acquire the user's position. Using an IrDA device instead of RFID tags, any special action but walking close to an IrDA marker is not required for the user.

In this paper, we propose a wearable annotation overlay system using an IrDA device and a passometer. First, the proposed system specifies the user's position exactly at appointed points where IrDA markers are set up to. The IrDA marker always send a beacon including its position ID. The IrDA receiver which the user equips receives the beacon and the system specifies the user's position when the user is in the infrared ray range of the IrDA marker. When the user recedes from the IrDA marker, the user's position is estimated by using a passometer which includes an electronic compass and acceleration sensors. The former can detect which direction the user walks and the latter can count how many steps the user walks. Moreover, an inertial sensor which is attached to the headset detects the orientation of the user's viewpoint. The system generates annotation overlay images from information such as the position, the orientation, and the database of annotations which is held in a wearable computer, and shows annotation overlay images to the user through the display device attached to the headset.

This paper is structured as follows. Section 2 describes the proposed wearable AR system using the IrDA device and the passometer. In Section 3, experimental results with a prototype system are described. Finally, Section 4 describes summary and future work.

\section{WEARABLE AUGMENTED REALITY SYSTEM}

AR systems need three kind of information to overlay annotations on the real world in the correct position: a position and an orientation of the user's viewpoint, and an annotation database including annotation information. The proposed system acquires the user's position using an IrDA device and a passometer. The system gets the orientation of the user's viewpoint using the inertial sensor attached to the user's headset. The annotation database is stored in a wearable computer. In Section 2.1, the hardware configuration of the proposed system is described. Section 2.2 describes how the system measures the user's position in detail. Section 2.3 describes the method of generating annotation overlay images.

\subsection{Hardware Configuration of Wearable Augmented Reality System}

Figure 2 shows the hardware configuration and data flow of the proposed wearable augmented reality system. The user equips four sensors, a notebook PC and a display device. Four sensors obtain the position and orientation of the user's viewpoint and the real scene image. These data are sent to a notebook PC. The notebook PC generates annotation overlay images from these data and a database of annotations which is stored in the notebook PC. The notebook PC sends annotation overlay images to a display device which is attached to the user's headset. The user can see it through the display device. Components of the proposed system are described in more detail in the following.

Sensors The user equips following four sensors.

Inertial sensor (Intersense: InterTrax ${ }^{2}$ ) It is attached to the user's glasses and measures the orientation of the user's viewpoint. It can transmit data at $256 \mathrm{~Hz}$ to a computer through USB connection. Electric power is supplied from the notebook PC through USB connection. 


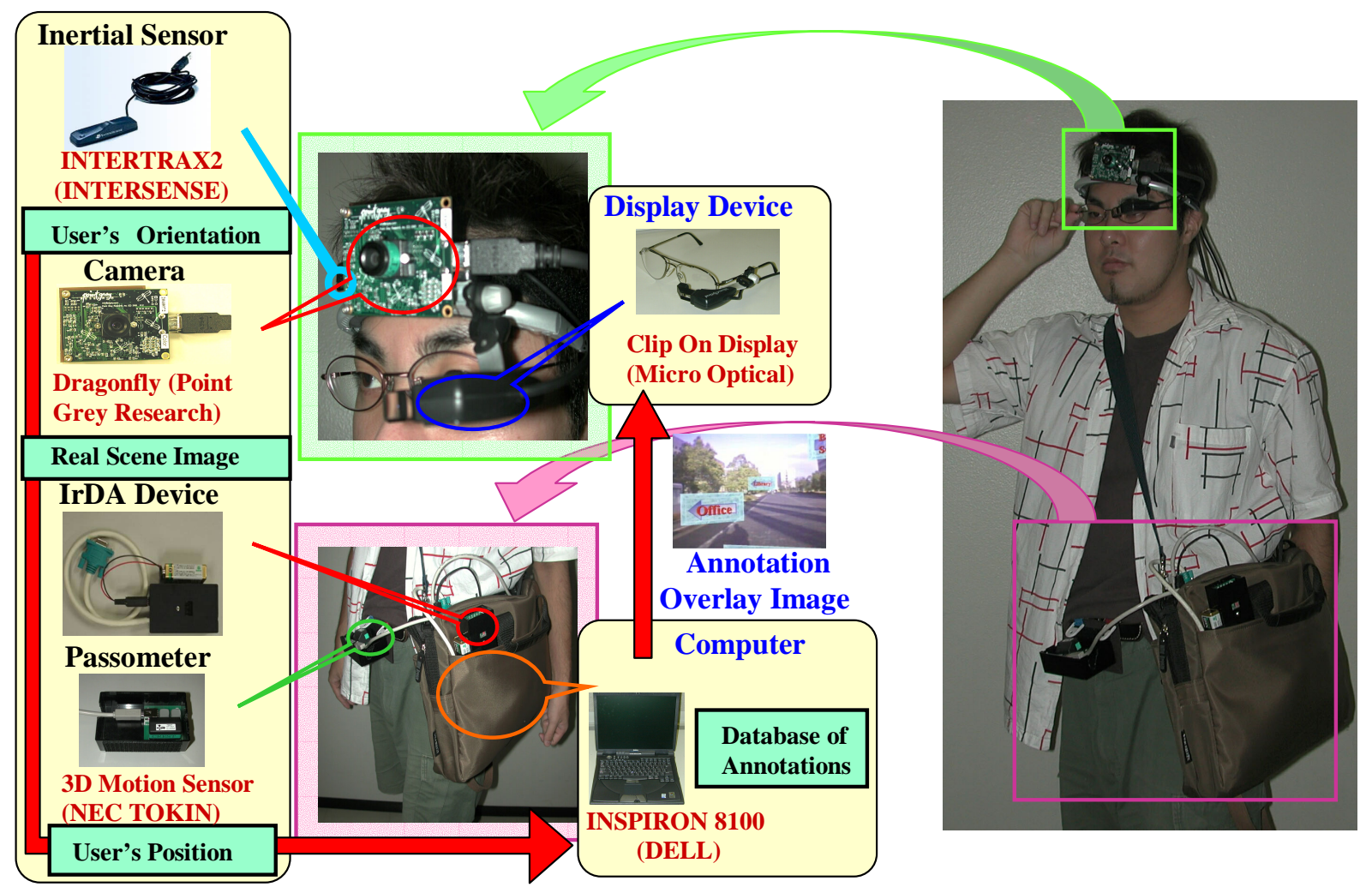

Figure 2. Hardware configuration and data flow of the proposed system.

Camera (Point Grey Research: Dragonfly) The camera is attached to the user's glasses and captures the real scene image from the user's viewpoint. It can capture RGB 24bit color image of $640 \times 480$ pixels. It can transmit the image 30 frames per second to a computer through IEEE1394 connection. Electric power is supplied from the notebook PC through IEEE1394 connection.

IrDA device (Original) An IrDA receiver is attached to the user's bag, and IrDA markers are set up at appointed points as shown in Figure 3 (c). The IrDA marker can send a beacon as far as about 5 meters. The IrDA markers always send the unique position ID. The IrDA receiver transmits the data through serial connection. Electric power of the receiver and the marker is supplied from a $9 \mathrm{~V}$ battery.

Passometer (NEC TOKIN: 3D motion sensor) A 3D motion sensor can measure pitch, roll, and yaw. It can also measure accelerations in the horizontal directions. It detects what direction and how many steps the user walks from the point where an IrDA marker is set up to. It can transmit data at $125 \mathrm{~Hz}$ to a computer through USB connection. Power is also supplied from the notebook PC through USB connection.

Computer (DELL: Inspiron8100, PentiumIII 1.2GHz, 512Mbytes memory) The notebook PC has a database of annotation information for generating annotation overlay images.

Display device (MicroOptical: Clip On Display) A small display device is attached to glasses as shown in Figure 2. The display device present a $640 \times 480$ color image to the user. Power is supplied from a control box which is carried in the user's shoulder bag. The user can see annotation overlay images through it; that is, the proposed system is a video see-through augmented reality system. 


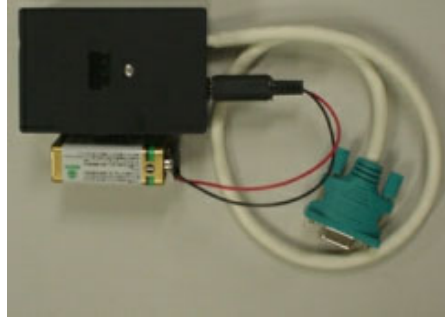

(a) IrDA receiver.

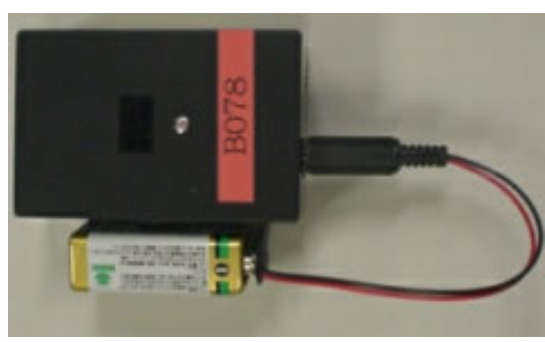

(b) IrDA marker.

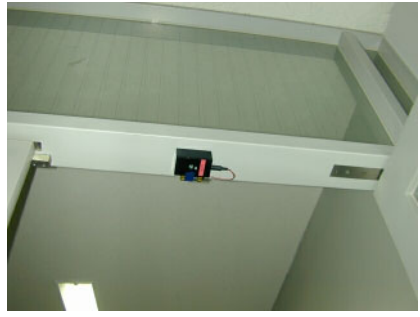

(c) IrDA marker set up at the ceiling of the door sector.

Figure 3. IrDA receiver and marker.

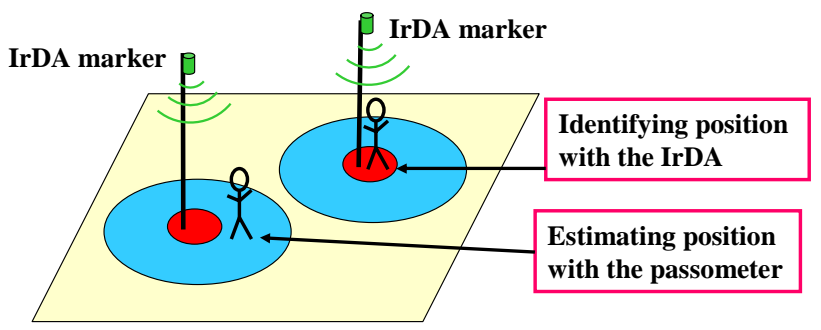

Figure 4. Method for measuring the user's position.

\subsection{Measurement of User's Position}

The proposed system measures the user's position indoors and outdoors using an IrDA device and a passometer as shown in Figure 4. The system identifies the user's accurate position using an IrDA device when the user is in the infrared ray range of IrDA markers. If the user goes out of the infrared ray range, the user's position is estimated by using a passometer. An accumulative error of the passometer is corrected when the user comes into the infrared ray range again. In the following, Section 2.2.1 describes the method for identifying the user's position using an IrDA device. Section 2.2.2 describes how the system estimates the user's position using a passometer.

\subsubsection{Identification of User's Position Using an IrDA device}

To specify the user's position at appointed points exactly, the system uses an IrDA device. Figure 3(a) shows an IrDA receiver, and (b) shows an IrDA marker. The IrDA markers are set up to appointed points as a positioning infrastructure. Figure 3(c) shows the IrDA marker set up at the ceiling of the door sector. IrDA markers always send their unique position IDs. On the other hand, the IrDA receiver is set up to the user's shoulder bag as shown in Figure 2. When the user comes into the infrared ray range of an IrDA marker, the user's position is identified by the position ID which is received from the IrDA marker. If it is difficult to set up IrDA markers constantly, IrDA markers should be set up only at the points where the user needs guiding information, such as at branch points or in front of guide boards.

\subsubsection{Estimation of User's Position Using a Passometer}

When the user goes out of infrared ray range, the user's position is estimated by the passometer. The passometer consists of a compass and acceleration sensors. The former can detect which direction the user walks toward. The latter can count how many steps the user walks. ${ }^{14,15}$ These data and the user's pace make it possible to estimate the user's position in the neighborhood of IrDA markers.

The proposed system regards the direction of the user's waist as the walking direction. When the user walks, the periodical pattern of the user's waist acceleration in the back-and-forth direction appears. The acceleration sensor of a passometer detects the pattern, and counts the number of user's steps. Figure 5 shows the typical 


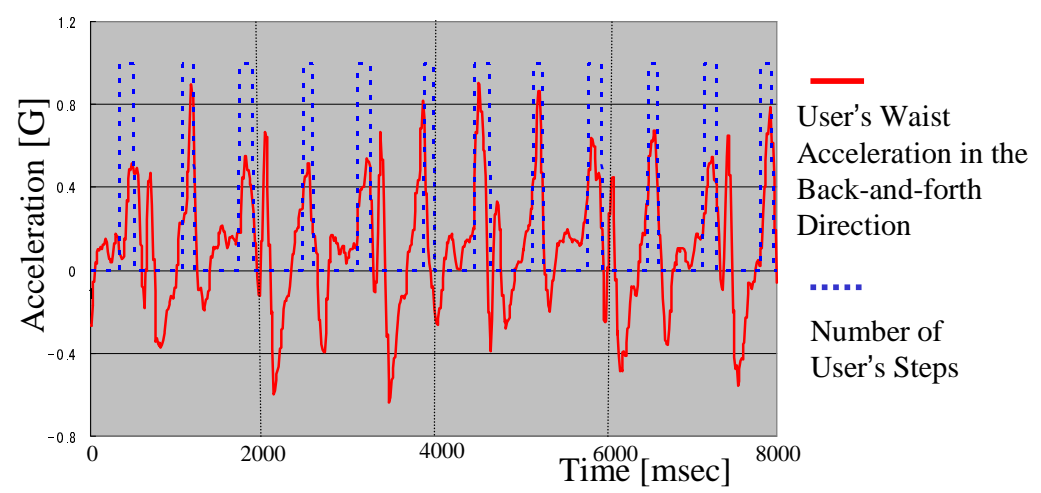

Figure 5. Relationship between the number of user's steps and the user's waist acceleration in the back-and-forth direction.

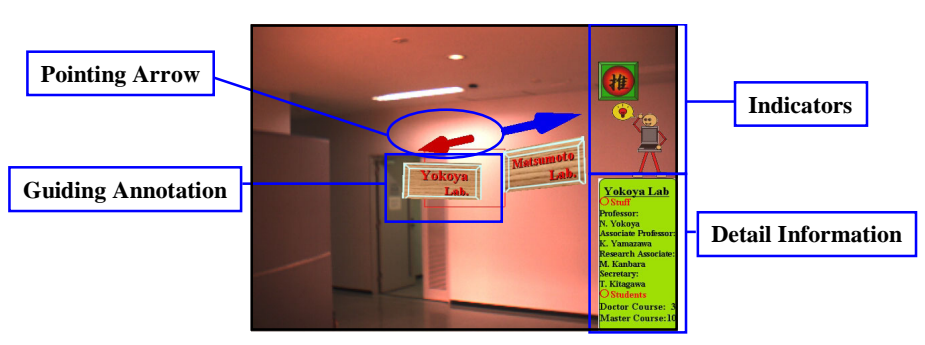

Figure 6. An example of annotation overlay image.

relationship between the number of user's steps and the user's waist acceleration in the back-and-forth direction. In Figure 5, a solid line shows the user's waist acceleration in the back-and-forth direction. A pair of ups and downs of dotted line means a user walk of one step. Note that the user's steps are manually measured. The graph indicates that the user's waist acceleration has a pair of a peak and a valley when the user walks one step. From Figure 5, the system can count the user's steps by counting the number of pairs of a peak and a valley in the user's waist acceleration pattern.

\subsection{Generation of Annotation Overlay Image}

As mentioned above, the system needs the database of annotations to generates annotation overlay images that are presented to the user. The database of annotations is consisted of the following data:

- Image files of guiding annotations and detail annotations,

- Directions of pointing arrows,

- Positions of annotations and IrDA markers.

The system selects appropriate annotations from this database according to the user's estimated position in the real world.

The proposed system generates an annotation overlay image from the user's position and the orientation of the user's viewpoint, and the database of annotations. Figure 6 shows an example of annotation overlay image. In our system, Annotations include four kinds of information: indicators, pointing arrows, guiding annotations, and detail annotations. Indicators show users a state of the system. Pointing arrows are used to link guiding annotations to the objects in the real scene. The guiding annotations are used for showing the names of the real 
objects to the user. The pointing arrow and the guiding annotation are placed in the appointed points in the real scene. The closer the user walks to a guiding annotation, the larger it is shown to the user. Therefore, the user can intuitively link guiding annotations to real objects. Detail annotations show the user detail information about the real object. A detail annotation appears on the lower right side of an image while the user notices the corresponding guiding annotations. The system recognizes that the user notices a guiding annotation when it is on the center of an annotation overlay image.

\section{EXPERIMENTS AND RESULTS}

\subsection{Experiments}

We have carried out some experiments using the proposed wearable augmented reality system. We have developed a navigation system which works in our campus where a number of IrDA markers are placed at appointed points. Sections 3.1.1 and 3.1.2 describe indoor and outdoor navigation experiments, respectively.

\subsubsection{Navigation in Indoor Environment}

Figure 7 illustrates an indoor experimental environment. In Figure 7, the points numbered P1, P2, and P3 mean the positions where the IrDA marker is set up. The user walked along the dotted arrows in Figure 7 from P1 to P3. Figure 8(a),...,f) are annotation overlay images presented to the user when the user was at the point (a),..,(f) in Figure 7, and the user's orientation was along the solid arrows at the point (a),..,(f) in Figure 7, respectively. Assuming the position $\mathrm{P} 1$ as an origin, a horizontal plane as the $\mathrm{x}-\mathrm{y}$ plane, and the $\mathrm{z}$ axis as a vertical direction as shown in Figure 7, the coordinates of the points P2, P3, and A, .., G are mentioned in Figure 7 (the unit of them is millimeter). Figure 8(a), (c), and (e) are the annotation overlay images when the system identified the user's position using IrDA devices and (b), (d), and (f) are ones when the system estimated the user's position using the passometer. The point (b) in Figure 7 is the position six steps away from the point (a), the point (d) is the position six steps away from the points (c), and the point (f) is the position four steps away from the point (e), respectively. Through this experiment, even if the user moves some steps in the indoor environment, the user can obtain annotations correctly overlaid on real scene images in real time.

\subsubsection{Navigation in Outdoor Environment}

Figure 9 illustrates an outdoor experimental environment. In Figure 9, the points numbered P1, P2, and P3 mean the positions where the IrDA marker is set up. The user walked along the dotted arrows in Figure 9 from P1 to P3. Figure 10(a),..,(f) show annotation overlay images when the user was at the point (a),...,f) in Figure 9, and the user's orientation was along the solid arrows at the point (a),...,(f) in Figure 9, respectively. Assuming the position $\mathrm{P} 1$ as an origin, a horizontal plane as the $\mathrm{x}-\mathrm{y}$ plane, and the $\mathrm{z}$ axis as a vertical direction as shown in Figure 9, the coordinates of the points P2, P3, and A, ..., G are mentioned in Figure 9 (the unit of them is millimeter). Figure 10(a), (c), and (e) are the annotation overlay images when the system identified the user's position using IrDA devices and (b), (d), and (f) in Figure 9 are ones when the system estimated the user's position using the passometer. The points (b), (d), and (f) are the positions eight steps away from the points (a), (c), and (e), respectively. Through this experiment, even if the user moves some steps in the outdoor environment, the user can obtain the images overlaid annotations correctly.

\subsection{Discussion}

The annotation overlay image can be presented to the user at 25fps. The weight of the user's headset is about $200 \mathrm{~g}$ and the user's shoulder bag which includes the notebook PC and batteries is about 5kg. The system can run with the battery more than two hours. Experimental results have shown the feasibility of the proposed system both indoors and outdoors.

As shown in (b) and (d) of Figure 10, when there exist occlusions among objects in the scene, the system may overlay wrong annotations. Therefore, the user cannot intuitively link annotations to the correct real objects. Using a simple 3D model of the real scene, we could represent annotations with correct occlusion. This should

further be investigated. 


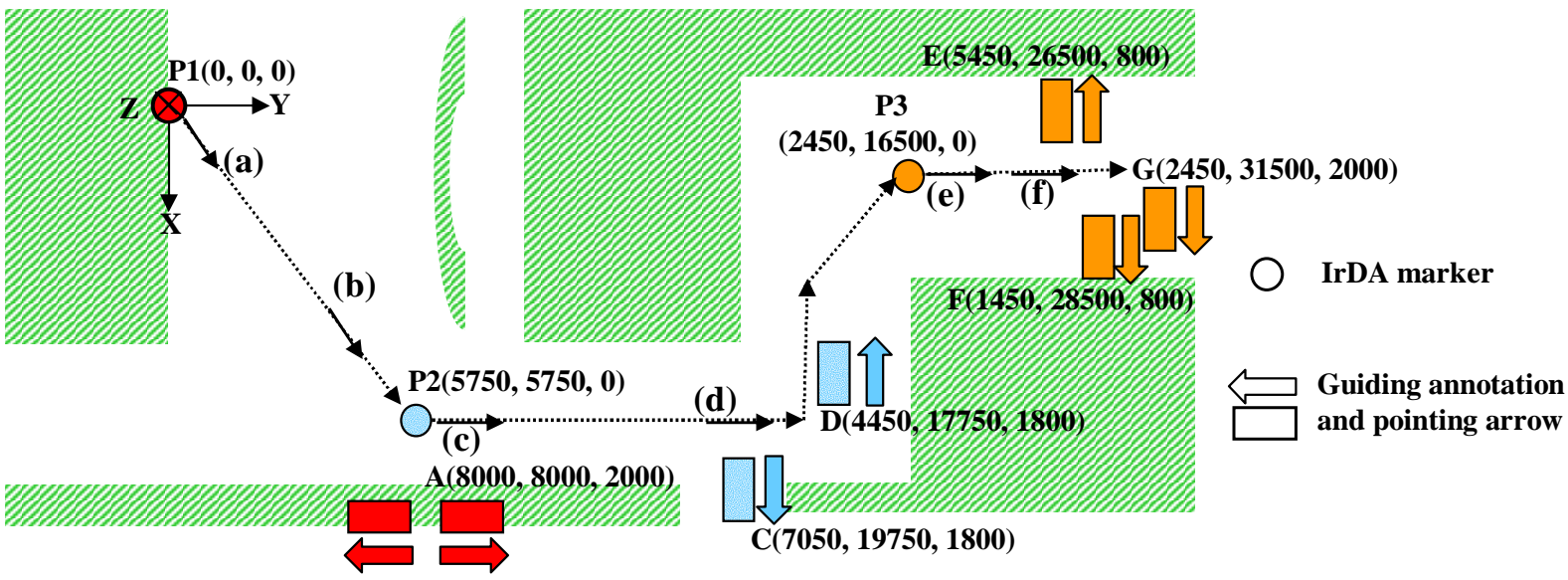

B(8000, 5000, 2000)

Figure 7. An indoor experimental environment.

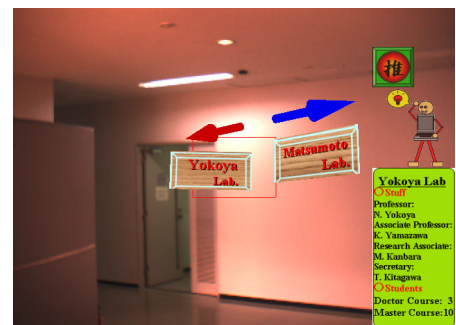

(a)

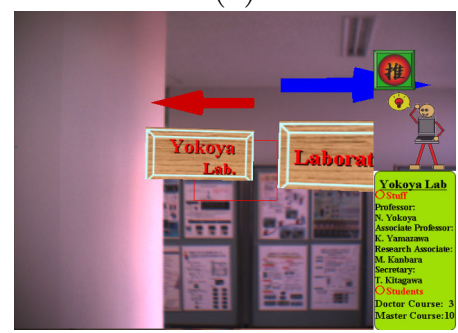

(d)

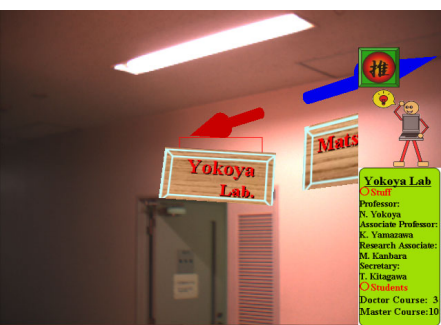

(b)

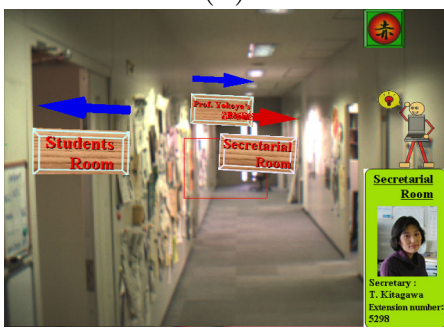

(e)

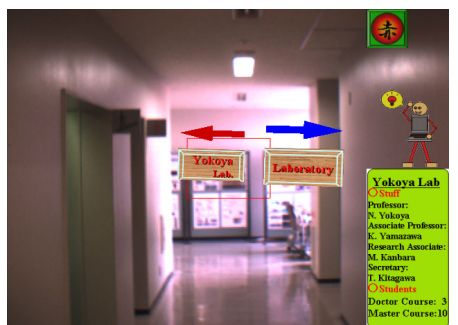

(c)

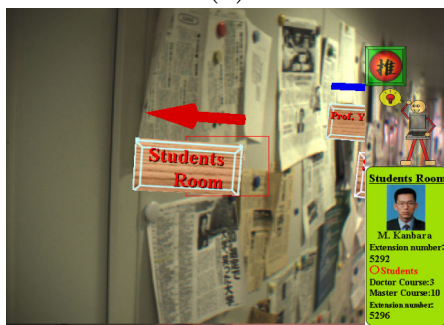

(f)

Figure 8. Examples of annotation overlay images.

\section{SUMMARY}

This paper has described a wearable augmented reality system which can be used in indoor and outdoor environments seamlessly using an IrDA device and a passometer. We have shown the feasibility of the proposed system demonstrating with experiments in both indoor and outdoor environments. In the future, we should use wireless LAN system to update annotation database automatically and solve the occlusion problem in presenting annotations.

\section{ACKNOWLEDGMENTS}

This research is partially supported by Core Research for Evolutional Science and Technology (CREST) Program "Advanced Media Technology for Everyday Living" of Japan Science and Technology Corporation (JST) and also by the Grant-in-Aid for Scientific Research from the Ministry of Education, Culture, Sports, Science and Technology. 

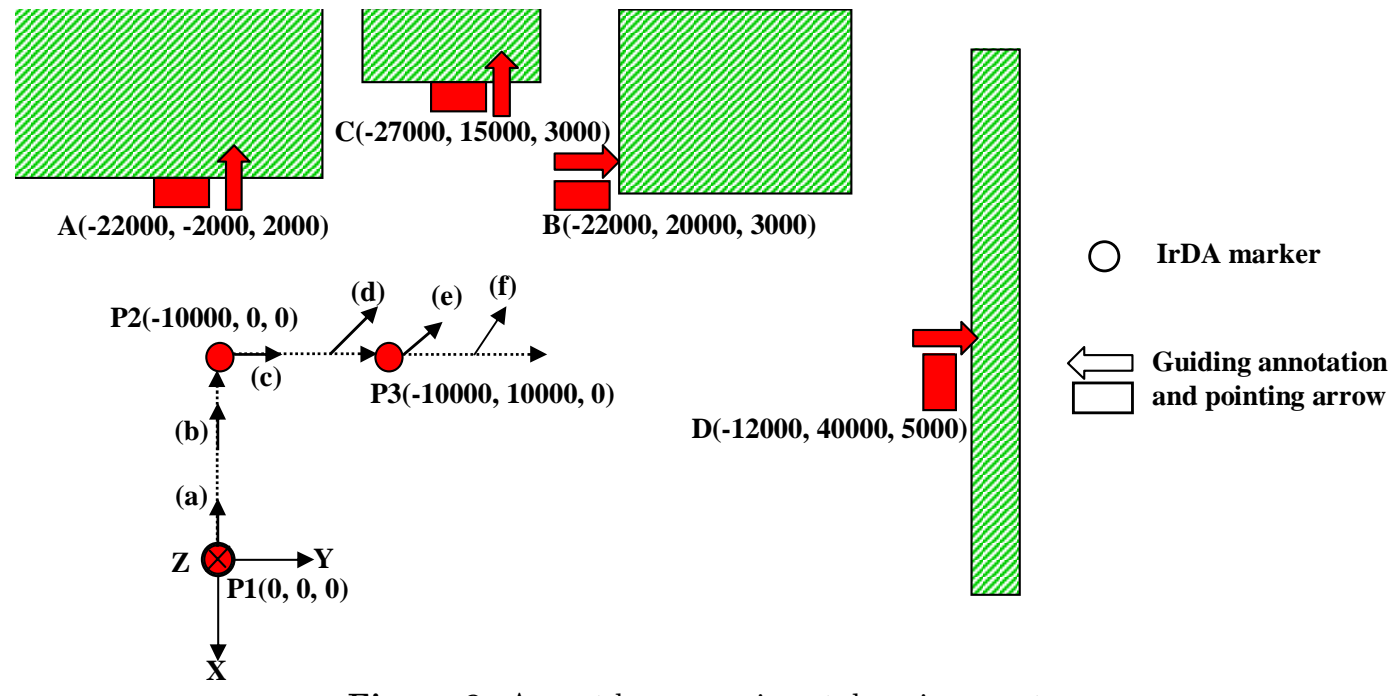

Figure 9. An outdoor experimental environment.

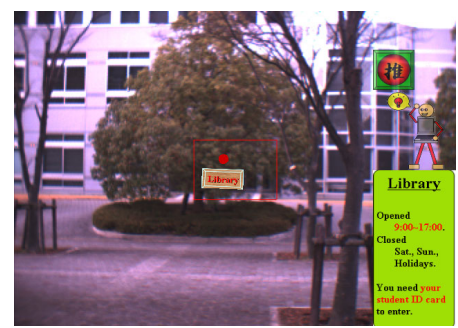

(a)

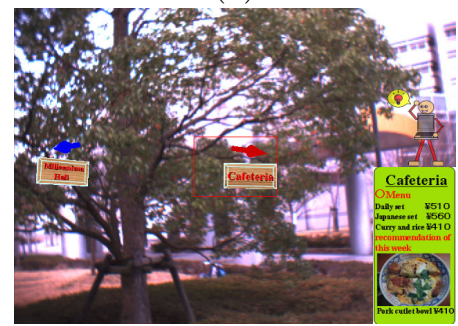

(d)

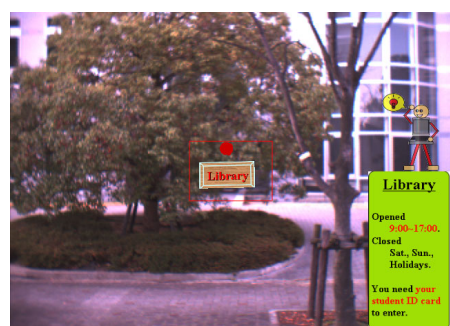

(b)

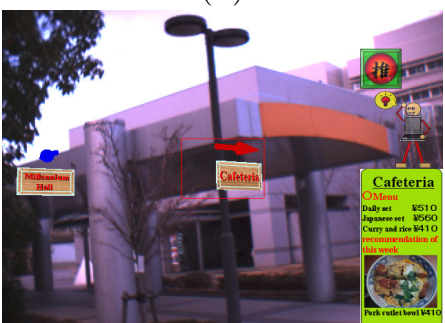

(e)

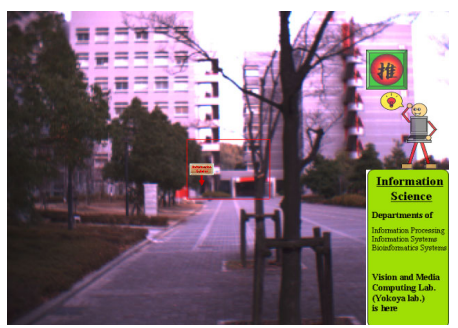

(c)

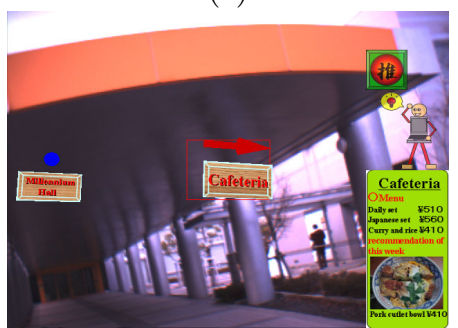

(f)

Figure 10. Examples the annotation overlay images.

\section{REFERENCES}

1. S. Mann: "Wearable Computing: A First Step Toward Personal Imaging," IEEE Computer, Vol. 30, No. 2, pp. 25-32, 2002.

2. R. Azuma: "A Survey of Augmented Reality," Presence, Vol. 6, No. 4, pp. 355-385, 1997.

3. M. Kanbara, T. Okuma, H. Takemura and N. Yokoya: "A Stereoscopic Video See-through Augmented Reality System Based on Real-time Vision-based Registration," Proc. IEEE Int. Conf. on Virtual Reality 2000, pp. 255-262, 2000.

4. S. Julier, M. Lanzagorta, Y. Baillot, L. Rosenblum, S. Feiner, T. Holler, and S. Sestito: "Information Filtering for Mobile Augmented Reality, " Proc. IEEE/ACM 1st Int. Symp. on Augmented Reality, pp. 3-11, 2000. 
5. S. Julier, M. Lanzagorta, Y. Baillot, L. Rosenblum, S. Feiner, T. Höller, and S. Sestito: "Personal Guidance System for the Visually Impaired," Proc. Int. Conf. on Assistive Technologies, pp. 85-90, 1994.

6. M. Kourogi, T. Kurata, and K. Sakaue: "A Panorama-based Method of Personal Positioning and Orientation and Its Real-time Applications for Wearable Computers," Proc. 5th Int. Symp. on Wearable Computers, pp. 107-114, 2001.

7. K. Satoh, K. Hara, M. Anabuki, H.Yamamoto, and H.Tamura: "TOWNWEAR: An Outdoor Wearable MR System with High-precision Registration," Proc. 2nd Int. Symp. on Mixed Reality, pp. 210-211, 2001.

8. M. Billinghurst, S. Weghorst, and T. Furness : "Wearable Computers for Three Dimensional CSCW," Proc. 1st Int. Symp. on Wearable Computers, pp. 39-46, 1997.

9. P. Daehne, J. Karigiannis: "ARCHEOGUIDE: System Architectureof a Mobile Outdoor Augmented Reality System," Proc. 1st Int. Symp. on Mixed and Augmented Reality, pp. 263-264, 2002.

10. H. Petrie, V. Johnson, T. Strothotte, A. Raab, S. Fritz, and R. Michel: "MoBIC: Designing a Travel Aid for Blind and Elderly People," Jour. of Navigation, Vol. 49, No. 1, pp. 44-52, 1996.

11. S. Feiner, B. MacIntyre, T. Höller, and A. Webster: "A Touring Machine: Prototyping 3D Mobile Augmented Reality Systems for Exploring the Urban Environment," Proc. 1st Int. Symp. on Wearable Computers, pp. 74-81, 1997.

12. A. State, G. Horita, D. Chen, W. Garrett, and M. Livingston: "Superior Augmented Reality Registration by Integrating Landmark Tracking and Magnetic Tracking," Proc. SIGGRAPH'96, pp. 429-438, 1996.

13. R. Tenmoku, M. Kanbara, N. Yokoya, and H. Takemura: "Annotation Overlay with a Wearable Computer Using Augmented Reality," Proc. 1st CREST Workshop on Advanced Computing and Communicating Techniques for Wearable Information Playing, pp. 27-32, 2002.

14. S. W. Lee and K. Mase: "A Personal Indoor Navigation Syatem Using Wearable Sensors," Proc. Int. Symp. on Mixed Reality, pp. 147-148, 2001.

15. S. W. Lee and K. Mase: "Incremental Motion-based Location Recognition," Proc. 5th Int. Symp. on Wearable Computers, pp. 123-130, 2001. 